\title{
Exhaled nitric oxide predicts control in patients with difficult-to-treat asthma
}

\author{
L.A. Pérez-de-Llano*, F. Carballada\#, O. Castro Añón*, M. Pizarro*, R. Golpe*, \\ A. Baloira", M. Vázquez Caruncho ${ }^{+}$and M. Boquete ${ }^{\#}$
}

ABSTRACT: We aimed to evaluate the accuracy of baseline exhaled nitric oxide fraction (FeNO) to recognise individuals with difficult-to-treat asthma who have the potential to achieve control with a guideline-based stepwise strategy.

102 consecutive patients with suboptimal asthma control underwent stepwise increase in the treatment with maximal fluticasone/salmeterol combination dose for 1 month. Then, those who remained uncontrolled received oral corticosteroids for an additional month.

With this approach, 53 patients $(52 \%)$ gained control. Those who achieved control were more likely to have positive skin results $(60.4 \%$ versus $34 \% ; p=0.01)$, positive bronchodilator test (57.1\% versus $35.8 \% ; p=0.02)$ and peak expiratory flow variability $\geqslant 20 \%(71.1 \%$ versus $49.1 \%$; $\mathbf{p}=\mathbf{0 . 0 4})$. Conversely, depression was more frequent in those who remained uncontrolled $(18.4 \%$ versus $43.4 \% ; p=0.01)$. An FeNO value $\geqslant 30 \mathrm{ppb}$ demonstrated a sensitivity of $87.5 \%(95 \% \mathrm{Cl} 73.9$ $94.5 \%)$ and a specificity of $90.6 \%(95 \% \mathrm{Cl} 79.7-95.9 \%)$ for the identification of responsive asthmatics.

The current results suggest that $F$ eNO can identify patients with difficult-to-treat asthma and the potential to respond to high doses of inhaled corticosteroids or systemic steroids.

KEYWORDS: Asthma control, difficult-to-treat, exhaled nitric oxide, salmeterol/fluticasone combination, severe asthma

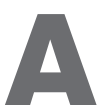
sthma treatment ideally achieves a steady state of no symptoms and no exacerbations with minimum medication. In practice, many patients with severe disease will not be optimally controlled, and asthma surveys indicate that a high proportion of patients remain uncontrolled even while receiving adequate therapy $[1,2]$. Despite guideline-based asthma management goals, the Asthma Insights and Reality in Europe (AIRE) study of 2,803 European patients showed that $46 \%$ of them reported daytime symptoms, 30\% had asthmarelated sleep disturbances at least once a week, $25 \%$ had unscheduled urgent care visit in the past year, $10 \%$ had an emergency room visit and $7 \%$ had an overnight hospitalisation [3].

There is no universally accepted definition of difficult-to-treat asthma. However, it is reasonable to consider it present when people have persistent symptoms and frequent exacerbations, despite being treated according to the Global Initiative for Asthma steps 4 or 5 . Patients with difficult-to-treat asthma are a cause of concern because of impairment of quality of life, continued decreased lung function and adverse effects of high-dose corticosteroids. Escalation of therapy to maximum doses of inhaled corticosteroids and oral corticosteroids is widely accepted in poorly controlled chronic asthmatics [4]. However, there is significant heterogeneity in the response to steroids and patients may be committed to an ineffective and potentially harmful therapy [5].

In the past years, asthma specialists have been interested in looking for an easy-to-measure and reliable biomarker that could facilitate the assessment of the disease and could avoid the risk of too little or too much treatment. Exhaled nitric oxide fraction (FeNO), an indirect marker of eosinophilic airway inflammation that is easy to perform and reproducible, has been successfully employed for the guidance of asthma management [6-8].

This study was designed to focus on the practical problem of the patient with diagnosed asthma whose symptoms are not controlled despite high
AFFILIATIONS

*Pneumology Service,

\#Allergology Division,

+Radiology Service, Hospital Xeral-

Calde, Lugo, and

'Respiratory Division, Hospital

Montecelo, Pontevedra, Spain.

CORRESPONDENCE

L.A. Pérez-de-Llano

Pneumology Service

Hospital Xeral-Calde

c/Dr Ochoa

s/n. 27004 Lugo

Spain

E-mail: eremos26@hotmail.com

Received:

July 262009

Accepted after revision:

Nov 172009

First published online:

Dec 082009 
doses of inhaled corticosteroids and other regular therapy. In this prospective, observational study, we adjusted medication according to a stepwise approach, corresponding to the international guidelines, based on asthma control. The aim was to evaluate the accuracy of baseline FeNO levels to recognise individuals with difficult-to-treat asthma who have the potential to achieve control with the stepwise approach. Control was defined as a score of $\geqslant 20$ in the Asthma Control Test (ACT). A secondary objective was to identify determinants of asthma control among variables related to clinical and functional patient characteristics.

\section{METHODS}

\section{Study design}

This was an observational and prospective study carried out in the pneumology and allergology units of the Hospital XeralCalde, Lugo, Spain. All patients were seen and assessed by the same physician at visit 1 and every subsequent clinical visit. The protocol was run on an outpatient basis.

\section{Patients}

Consecutive patients were recruited for protocol evaluation between April 2008 and July 2009 if they had a difficult-to-treat asthma defined as not controlled asthma (ACT score <20) despite minimal maintenance therapy of long-acting $\beta_{2}$-agonist and high-dose inhaled corticosteroids $(\geqslant 800 \mu \mathrm{g}$ beclometasone equivalent) for $>3$ months. All subjects had been free of exacerbations for at least this period. All of them were regularly followed at an outpatient asthma clinic and they were educated on the nature of the illness and the correct use of inhalers. The patients were advised to bring their maintenance mediation to the hospital and all of them denied nonadherence at the time of the first clinical assessment. Patients treated with oral corticosteroids or omalizumab were excluded. We also excluded those with a smoking history in excess of 10 pack-yrs. Inclusion criteria were relatively broad because the authors wanted to reflect usual clinical practice.

\section{Protocol}

First visit

Patients were classified according to their clinical control (assessed by the ACT). In all cases, medical history, spirometry, bronchodilator test, methacholine test (if not contraindicated), FeNO, and ambulatory peak expiratory flow (PEF) measurement were recorded. A detailed structured clinical history was obtained for all subjects in order to rule out comorbid conditions (obesity, smoking, anxiety, depression, medications, oesophageal reflux, upper airways disease, vocal cord dysfunction, bronchiectasis, chronic obstructive pulmonary disease (COPD) and allergic bronchopulmonary aspergillosis) that could influence asthma control. Vocal chord dysfunction was diagnosed with endoscopy when clinically suspected. Oesophageal reflux was diagnosed by means of medical history ( $\mathrm{pH}$ metering was not systematically performed). Patients with suspicion of bronchiectasis underwent high-resolution computed tomography. Anxiety and depression were assessed by use of Goldberg's short questionnaire. Allergic bronchopulmonary aspergillosis was investigated in patients with high immunoglobulin E levels.

Patients with suboptimal asthma control (ACT score $<20$ ) with correct inhalation technique received increased therapy with maximal fluticasone/salmeterol combination dose (unless they were treated with such medication) and they were placed to return for a second visit 1 month later.

\section{Second visit}

Patients with previous suboptimal asthma control who received increased therapy were assessed for clinical control (via ACT). If they achieved asthma control, new spirometry and FeNO measurements were performed. If they did not achieve control, they underwent another increase of asthma therapy with $30 \mathrm{mg}$ of deflazacort added to their inhaled medication and were placed to return for a third visit 1 month later.

\section{Third visit}

Patients were assessed for asthma control. New spirometry and $F$ eNO measurements were recorded.

The time course of the protocol is shown in figure 1 .

\section{Measurements}

Exhaled nitric oxide

A single measurement was undertaken using a portable device (NIOX MINO; Aerocrine AB, Solna, Sweden) at a mouth flow rate of $50 \mathrm{~mL} \cdot \mathrm{s}^{-1}$ for $10 \mathrm{~s}$. The sensor on the device was changed periodically, in line with the manufacturer's guidance.

\section{Pulmonary function test}

Spirometry was performed using a Datospir 120 spirometer (Sibelmed, Barcelona, Spain) in accordance with American Thoracic Society (ATS)/European Respiratory Society (ERS) guidelines [9] to determine forced expiratory volume in $1 \mathrm{~s}$ (FEV1), forced vital capacity (FVC), FEV1/FVC and PEF. FEV1 and FVC values are expressed as a percentage of predicted value, and changes in FEV1 are expressed as a percentage of

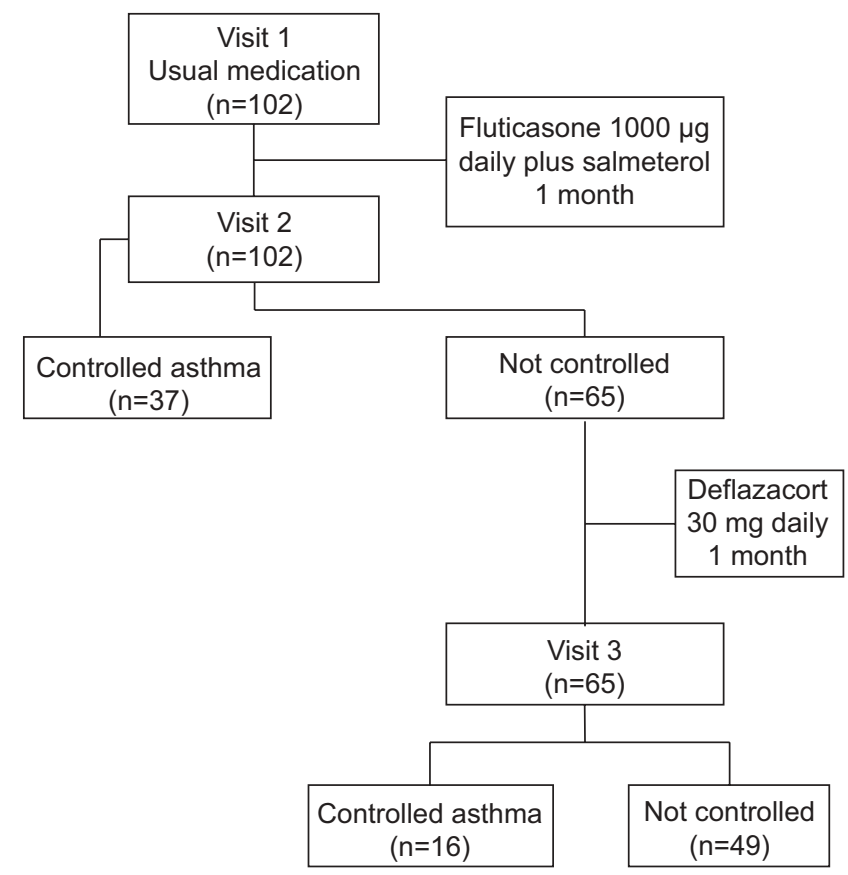

FIGURE 1. Flow chart summarising the study protocol. 
the initial value. Bronchodilator reversibility was calculated as the percentage of change in FEV1 from baseline, 15 min after inhaling $400 \mu \mathrm{g}$ of albuterol.

Airway hyperresponsiveness to methacholine (expressed as the provocative concentration causing a $20 \%$ decrease in FEV1 (PC20) was measured with a 2-min tidal breathing method adapted from CоскСROFT et al. [10]. After an initial nebulised saline challenge, subjects inhaled doubling concentrations, ranging from 0.038 to $19.2 \mathrm{mg} \cdot \mathrm{mL}^{-1}$ of methacholine bromide, at 5-min intervals. Airway hyperresponsiveness to methacholine was defined as a $\mathrm{PC} 20 \leqslant 8 \mathrm{mg} \cdot \mathrm{mL}^{-1}$.

Diurnal peak expiratory flow rate (PEFR) was recorded in a daily record card.

\section{Symptom assessment}

Asthma control was assessed using the validated Spanish version of the ACT [11]. Patients subjectively evaluated the degree of impairment caused by their asthma during the preceding 4 weeks by responding to five questions using a five-point scale. The ACT is reliable, valid and responsive to changes in asthma control over time. A cut-off score of $\leqslant 19$ identifies patients with poorly controlled asthma [12].

\section{Goldberg's anxiety and depression test}

This test was designed to be used by non-psychiatrists in clinical investigations. The score is based on responses of "yes" or "no" to nine depression and nine anxiety items (the full set of nine questions need to be administered only if there are positive answers to the first four), asking how respondents have been feeling in the past month. GOLDBERG et al. [13] considered patients with anxiety scores of $\geqslant 5$ or with depression scores of $\geqslant 2$ as having a $50 \%$ chance of a clinically important disturbance.

\section{Statistical analyses}

The baseline characteristics were compared between those who responded to increase of treatment with those who did not achieve control. Comparison of continuous data was made by t-test or Wilcoxon signed rank test, as appropriate. Unpaired t-test was used to compare means between groups and the paired test was employed when repeated measurements were made. Intergroup differences were evaluated by Chi-squared analysis or Fisher's exact test for categorical data.

In order to determine the concentration of FeNO capable of predicting asthma control after stepwise increase of therapy, we calculated sensitivity, specificity, positive predictive power and negative predictive power, with their 95\% confidence intervals, for each FeNO measurement. With the resulting data, we generated a receiver operating characteristic (ROC) curve to find the best FeNO cut-off (value with the greatest sensitivity and specificity) and to calculate the area under the curve (AUC), with its 95\% confidence interval, to estimate overall diagnostic accuracy. AUCs with a value close to 1 indicated excellent ability to discriminate. A positive likelihood ratio was calculated as sensitivity/(1-specificity) or true-positive rate/ false-positive rate. A positive likelihood ratio reflects increased odds of achieving control after a positive FeNO result. A negative likelihood ratio is (1-sensitivity)/specificity or falsenegative rate/true-negative rate and reflects reduced odds of achieving asthma control after a negative FeNO result. The ROC was fitted using the maximum likelihood fit of a binormal model by employing the web-based calculator JROCFIT [14].

Logistic regression models were used to assess the relationship between predictor variables (duration of the disease, positive reactions to skin-prick tests for common aeroallergens, clinical manifestations of atopic disease, presence of comorbidity, depression, body mass index (BMI), FeNO, FEV1 and daily PEF variability of $\geqslant 20 \%$ ) and the event of interest (response to therapy).

A value of $\mathrm{p}<0.05$ was considered significant.

Statistical analyses were made using the $\mathrm{R}$ statistical package (R Development Core Team).

\section{RESULTS}

\section{Patient demographics}

A total of 102 consecutive patients (mean \pm SD age $56 \pm 15$ yrs, $71.6 \%$ females) with difficult-to-treat asthma were prospectively included. At entry, mean ACT score was $14 \pm 1$. Baseline demographic and clinical characteristics are displayed in table 1.

Mean FEV1 at visit 1 was $72 \%$ pred and $50 \%$ of the patients showed an obstructive pattern. Baseline FeNO levels were $43.1 \pm 45.6 \mathrm{ppb}$ (range 4-222 ppb). Pulmonary function test results are summarised in table 2 .

Consistent with the inclusion criteria, all patients were taking a combination of high-dose inhaled corticosteroid and longacting $\beta_{2}$-agonist. A small proportion of the patients used additional supplementary treatments (table 3 ).

\section{Response to the stepwise increase of therapy}

At visit two, 37 patients $(36.2 \%)$ achieved control with the maximum dose of fluticasone/salmeterol combination. Of the remaining 65 patients, $16(24.6 \%)$ gained control after 1 month of oral steroids (fig. 1).

Only one patient suffered an exacerbation that required oral steroids, but not hospitalisation, during the study. Another one was diagnosed with pneumonia (and admitted to hospital for this reason) while taking oral steroids between the second and third visit.

Baseline characteristics for the two groups (patients who achieved control and those who did not) are listed in table 1. Those patients who achieved control were more likely to have positive skin results $(60.4 \%$ versus $34 \%$; $p=0.01)$, positive bronchodilator test $(57.1 \%$ versus $35.8 \% ; \mathrm{p}=0.02)$ and PEF variability of $>20 \%$ ( $71.1 \%$ versus $49.1 \%$; $p=0.04)$. Conversely, depression was more frequent in the group of patients who remained uncontrolled ( $18.4 \%$ versus $43.4 \%$; $\mathrm{p}=0.01$ ).

After applying the approach mentioned above, we have found that FEV1 increased from $1.9 \pm 0.7 \mathrm{~L} \cdot \mathrm{min}^{-1}$ to $2.1 \pm 0.8 \mathrm{~L} \cdot \mathrm{min}^{-1}$ $(\mathrm{p}<0.01)$, ACT increased from 14 (range 7-19) to 19 (7-25) $(\mathrm{p}<0.01)$ and FeNO levels decreased from $28(4-222)$ to 17.5 $(4-94)(\mathrm{p}<0.01)$.

At baseline, FeNO was $67 \pm 49 \mathrm{ppb}$ in patients who finally achieved control, whereas it was $28 \pm 36 \mathrm{ppb}$ in those who did not gain control $(\mathrm{p}<0.0001)$. At the end of the study, FeNO was 
TABLE 1 Study subjects' baseline demographic and clinical characteristics by asthma control

\begin{tabular}{|c|c|c|c|c|}
\hline & All subjects & Controlled & Uncontrolled & Controlled versus uncontrolled \\
\hline Age yrs & $56 \pm 15$ & $53 \pm 17$ & $58 \pm 13$ & $\mathrm{p}=0.11$ (NS) \\
\hline $\mathrm{BMI} \mathbf{k g} \cdot \mathrm{m}^{-2}$ & $29.04 \pm 6$ & $27.95 \pm 6.58$ & $29.66 \pm 5.37$ & $\mathrm{p}=0.16$ (NS) \\
\hline Current smoker & 7 (6.9) & $3(6.1)$ & $4(7.5)$ & $\mathrm{p}=0.72(\mathrm{NS})$ \\
\hline Duration of the disease yrs & $20 \pm 15$ & $19 \pm 14$ & $25 \pm 16$ & $\mathrm{p}=0.07$ (NS) \\
\hline Adult & $82(80.2)$ & $40(79.6)$ & $42(81.8)$ & \\
\hline Positive skin test & $47(46.5)$ & $29(60.4)$ & $18(34)$ & $p=0.01$ \\
\hline Allergic comorbidities & $70(69.3)$ & $35(71.4)$ & $35(67.3)$ & $\mathrm{p}=0.67(\mathrm{NS})$ \\
\hline Aspirin intolerance & $8(8)$ & $2(5.0)$ & $6(11.8)$ & $\mathrm{p}=0.26$ (NS) \\
\hline Polyposis & $9(8.8)$ & $5(12.5)$ & $4(7.5)$ & $\mathrm{p}=0.42(\mathrm{NS})$ \\
\hline ABPA & $10(9.8)$ & $4(8.2)$ & $6(11.3)$ & $\mathrm{p}=0.74(\mathrm{NS})$ \\
\hline Depression & $32(31.4)$ & $9(18.4)$ & $23(43.4)$ & $p=0.01$ \\
\hline ACT score & $14 \pm 1$ & $14 \pm 3$ & $12 \pm 3$ & $p<0.01$ \\
\hline
\end{tabular}

Data are presented as $n(\%)$ or mean \pm SD, unless otherwise stated. BMI: body mass index; ABPA: allergic bronchopulmonary aspergillosis; ACT: Asthma Control Test; NS: nonsignificant.

$32 \pm 21 \mathrm{ppb}$ in patients who achieved control and $16 \pm 12 \mathrm{ppb}$ in those who did not $(\mathrm{p}<0.0001)$. Interestingly, FeNO levels were above $35 \mathrm{ppb}$ in $25 \%$ of the patients who gained control at the final visit.

Figure 2 shows the change in the median of ACT score and FeNO value over time.

\section{Predictive utility of FenO}

FeNO was an excellent marker for predicting therapeutic response, with an ROC AUC of 0.925 . A FeNO value $>30 \mathrm{ppb}$ demonstrated a sensitivity of $87.5 \%$ (95\% CI $73.9-94.5 \%)$ and a specificity of $90.6 \%$ (95\% CI 79.7-95.9\%) for the identification of patients who will achieve control (fig. 3). The negative predictive value was $90.6 \%$ (95\% CI $79.7-95.9 \%)$, and the positive predictive value was $87.5 \%$ (95\% CI $73.9-94.5 \%)$. With a cut-off value of $30 \mathrm{ppb}$ for FeNO, the positive likelihood ratio was 9.3 (95\% CI 3.9-21.5) and the negative likelihood ratio was 0.14 (95\% CI 0.06 to 0.32$)$.

Sensitivity, specificity, likelihood ratios and positive and negative predictive values at different cut-off points of FeNO values are shown in table 4

\section{Factors associated with achievement of control}

On the basis of the univariate analysis, the following variables were introduced into the multivariate model: $\mathrm{FeNO}$, presence of comorbidity, positive skin-prick testing, duration of the disease, BMI, FEV1, PEF variability $>20 \%$ and depression. The multivariate analysis showed that only FeNO (OR 47.7, 95\% CI 13.9163.9) independently and significantly correlated with the achievement of control.

\section{TABLE 2 Pulmonary function test}

All subjects

FEV $1 \%$ pred

FEV $1 /$ FVC $<70 \%$

Positive bronchodilator test

PEF variability $>\mathbf{2 0} \%$

Positive methacholine test

FeNO ppb

$\begin{array}{ccc}72 \pm 24 & 75 \pm 20 & 69 \pm 27 \\ 51(50) & 25(49) & 26(51) \\ 47 / 99 & 30(57.1) & 17(35.8) \\ 58(59) & 32(71.1) & 26(49.1) \\ 69 / 71 & 35(72.9) & 34(66.7) \\ 43.1(4-222) & 68.4(11-222) & 19.7(4-160)\end{array}$

Data are presented as $n, n(\%)$, mean \pm SD or median (range), unless otherwise stated. FEV1: forced expiratory volume in 1 s; \% pred: \% predicted; FVC: forced vital capacity; PEF: peak expiratory flow; FeNO: exhaled nitric oxide fraction; NS: nonsignificant. 


\begin{tabular}{lc}
\hline TABLE $\mathbf{3}$ & Treatment at baseline \\
\hline Treatment & Patients \\
\hline BF $(\mathbf{6 4 0 / 9} \boldsymbol{\mu g})$ & $44(43.1)$ \\
FS $(\mathbf{1 0 0 0} / \mathbf{1 0 0} \boldsymbol{\mu g})$ & $21(20.5)$ \\
FS $(\mathbf{5 0 0} / \mathbf{1 0 0} \boldsymbol{\mu g})$ & $27(26.4)$ \\
Tiotropium bromide & $7(6.8)$ \\
Montelukast & $39(38.2)$ \\
Theophyline & $3(2.9)$ \\
\hline & \\
Data are presented as $\mathrm{n}(\%)$. BF: budesonide/formoterol daily maintenance \\
dose; FS: fluticasone/salmeterol daily maintenance dose.
\end{tabular}

\section{DISCUSSION}

Our results demonstrate, for the first time, that FeNO levels might be predictive of response to a stepwise approach in patients with difficult-to-treat asthma. This study adds to previous research showing a clinical utility of FeNO measurements in asthmatic patients [6-8]. It has been demonstrated that $\mathrm{FeNO}$ correlates with eosinophilic inflammation measured using bronchial biopsies and induced sputum [15, 16]. In addition, previous studies have shown that high numbers of sputum eosinophils were predictive of steroid response [17, 18]. This underlines that steroid response is related to particular characteristics of airway inflammation. Conversely, FeNO is reduced by treatment with inhaled corticosteroids [19], but elevated levels of this biomarker were previously observed in patients with severe asthma despite corticosteroid treatment [20]. This might imply either steroid resistant inflammatory processes in the airway, or insufficient doses of anti-inflammatory medication. Theoretically, FeNO measurements might help us to identify individuals with persistent eosinophilic inflammation in which a steroid response is more likely. This hypothesis is supported by our results in a difficult asthma population, indirectly by those of SМाTH et al. [8], who found that FeNO measurements provided a means of predicting steroid response in patients with undiagnosed respiratory symptoms, and also by the findings of LiTTLE et al. [21], who have shown that response to oral steroids in asthma patients can be predicted in most cases by analysing this biomarker. Even with various expert-derived guidelines that provide asthma treatment strategies, many patients remain suboptimally controlled. In our series, $48 \%$ of the patients did not achieve control, as assessed by the ACT questionnaire, despite receiving the best available treatment and optimal management efforts. This figure is in accordance with the Gaining Optimal Asthma Control (GOAL) study, which showed that symptoms were uncontrolled in as many as $38 \%$ of patients with moderate-tosevere asthma, despite high doses of salmeterol/fluticasone, good adherence (virtually 100\%) and tightly monitored inhalation techniques [22]. The addition of oral prednisolone $\left(0.5 \mathrm{mg} \cdot \mathrm{kg}^{-1}\right)$ led to a modest $7 \%$ increase in the percentage of well-controlled patients [22]. The most widely accepted explanation for these unsatisfactory findings is the view that the term "difficult-to-treat asthma" might include a broad spectrum of inflammatory patterns, not always as responsive to steroids as an eosinophil-associated process could be. In fact, several phenotypes of refractory asthma have been proposed, including
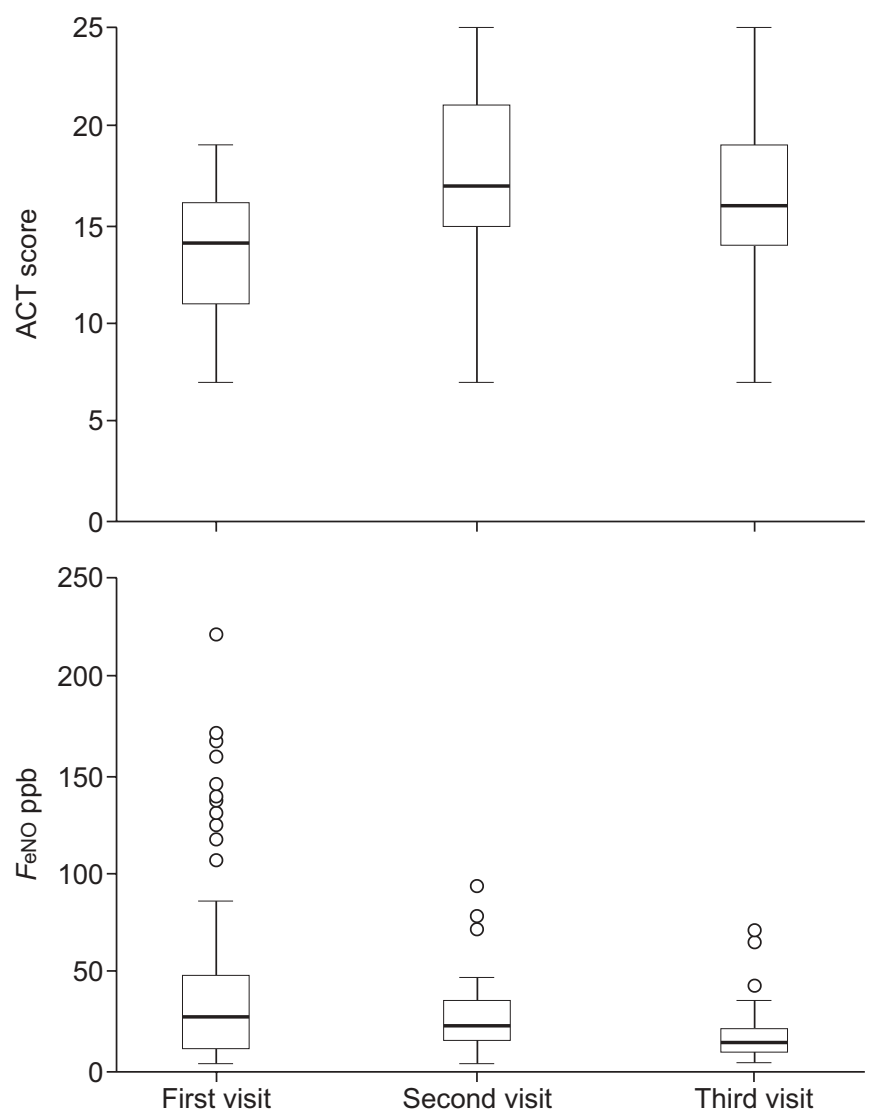

FIGURE 2. Box plots of Asthma Control Test (ACT) score and exhaled nitric oxide fraction $(\mathrm{FeNO})$ values in successive visits. Boxes represent median and interquartile range values; whiskers extend to fifth and 95th percentiles; outliers are shown individually. ACT score increased significantly from visit one to visit two. FeNO values decreased significantly from visit one to visit three. It must be noted that median ACT score was higher at visit two than at visit three. This apparent contradiction is explained by the fact that many patients achieved control (ACT score $>20$ ) at visit two. However, at visit three, the majority of subjects remained uncontrolled (ACT score <20). Conversely, median FeNO decreased at every visit, reflecting the fact that patients who did not achieve control showed low values of the biomarker.

those subjects who have persistent eosinophilic inflammation despite steroid treatment, but also those with predominant neutrophilic airway inflammation and those in whom virtually no inflammation is present on bronchial biopsy [23].

We have found a higher proportion of positive skin test results in those patients who achieved control than in those who remained uncontrolled. Although the classification between atopic and nonatopic disease has recently come under scrutiny, the ENFUMOSA (European Network for Understanding Mechanisms of Severe Asthma) study found fewer positive skin-prick tests in severe asthmatics compared with controlled patients, suggesting an association between atopy and the potential for poor/good asthma control with steroid/ $\beta$-agonist therapy [24]. Positive bronchodilator test and PEF variability $>20 \%$ were also significantly more common in asthmatics who gained control, possibly reflecting a more reversible clinical situation. Conversely, depression was more frequent in patients who did not achieve control. 


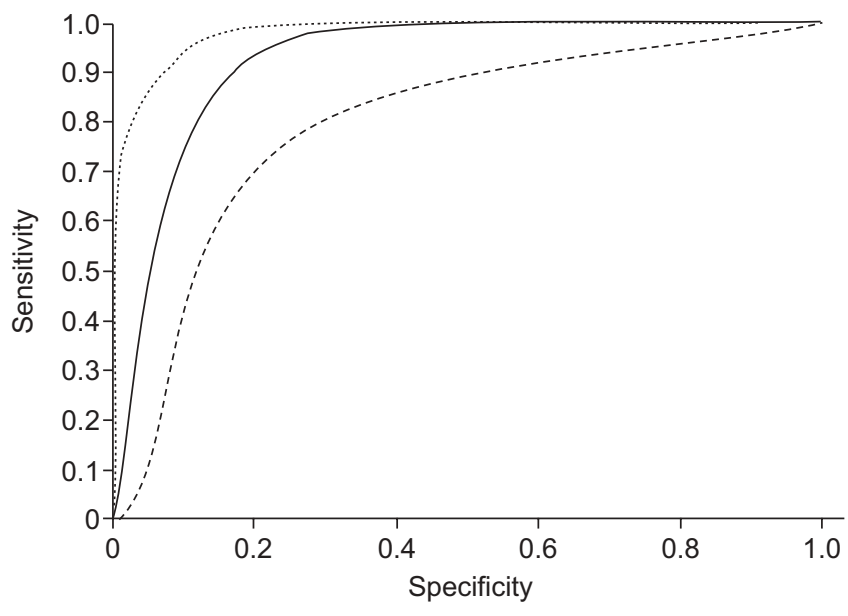

FIGURE 3. Receiver operating characteristic (ROC) curve for the prediction of therapeutic response from exhaled nitric oxide fraction ( $\left.F_{\mathrm{eNO}}\right)$ measurements. ROC curve identified the optimal cut-off value of $30 \mathrm{ppb}$ with $87.5 \%$ sensitivity $(95 \% \mathrm{Cl}$ 73.9-94.5\%) and 90.6\% specificity (95\% Cl 79.7-95.9\%). Area under the ROC curve is 0.925 . Broken lines illustrate the $95 \%$ confidence limits.

It must be noted that we assessed asthma control by administering the ACT. The recently published ATS/ERS consensus about standardisation of outcomes relating to asthma control recommend this kind of composite measure designed to provide numerical comparisons of treatment effect [25]. This brief five-item questionnaire measures several different areas of asthma control, including symptoms, rescue inhaler usage and the impact of asthma in everyday functioning, but, even using this tool, accurate assessment can be difficult and comorbidities might alter the scoring [26]. In two case series, coexisting disorders with asthma-like symptoms were found in $19 \%$ and $34 \%$ of patients with difficult asthma $[27,28]$. In such individuals, a variety of comorbid diseases, such as gastro-oesophageal reflux disease, obesity, vocal cord dysfunction and upper airway disease (e.g. seasonal allergies), may overlap with symptoms of asthma, making it difficult to assess control. Particularly, it has been reported that depressive and anxiety disorders were associated with a decreased level of asthma control, including more visits to the doctor or emergency room, inability to do usual activities, and more days of symptoms compared to those without depression or anxiety [29]. We have not found differences in the rate of other comorbid conditions between patients who reached control and those who remained uncontrolled.

Some limitations of this study must be addressed. 1) The sample size was small. 2) It is possible that the treatment periods (1 month) were too short to reach the maximum effect. In fact, one study demonstrated that asthmatic patients with stable dosing tend to improve further, confirming the benefit of sustained treatment in subjects who have difficulty in achieving control [22]. 3) Airway hyperresponsiveness, a factor that could potentially predict therapeutic response, was not assessed in all of the patients. 4) A selection bias is possible because the study design excluded patients who were taking oral steroids. Thus, the sample might not accurately represent the whole population of difficult-to-treat asthmatics. Conversely, it must be taken into account that the diagnosis of severe asthma still represents a challenge for physicians, and many patients with other entities like COPD could be categorised as "difficult-to-treat asthma". However, in our sample there were $71 \%$ females, more than $90 \%$ were never smokers, hyperresponsiveness was present in almost all of them and mean FeNO value was 43 ppb. All of these facts, taken together, make us feel confident that our patients were truly asthmatics. 5) The investigators were not blinded to the FeNO results. This fact could be a possible source of bias, although we believe that the influence in our results is not relevant because therapeutic decisions were not based on FeNO values, but rather were derived from the ACT score, which is self completed by the patients. 6) Although all of the patients had been regularly followed at an outpatient asthma clinic, they had previously been educated on the correct use of inhalers, they were advised to bring their maintenance mediation to the hospital and all of them denied nonadherence at the time of the first clinical assessment we did not measure adherence objectively. GAMBLE et al. [30] have recently demonstrated that a significant proportion of patients with difficult-to-control asthma remained nonadherent to inhaled or oral corticosteroids. However, patients were unaware that they were being observed and it is well known that patients who agree to participate in research are more likely than nonparticipants to be adherent with their regimen. Anyway, although noncompliance could underestimate the population response to the stepwise approach, it is unlikely to affect the predictive accuracy of FeNO.

\begin{tabular}{|c|c|c|c|c|c|c|}
\hline FeNO ppb & Sensitivity \% & Specificity \% & Positive LR & Negative LR & PPV & NPV \\
\hline 20 & 90 (76.9-96.0) & $81.1(68.6-89.4)$ & $4.77(2.70-8.42)$ & $0.12(0.05-0,32)$ & $78.3(64.4-87.7)$ & $91.5(80.1-96.6)$ \\
\hline 25 & 90 (76.9-96.0) & $84.9(72.9-92.1)$ & $5.96(3.12-11.39)$ & $0.12(0.05-0.30)$ & $81.8(68.0-90.5)$ & $91.8(80.8-96.8)$ \\
\hline 30 & $87.5(73.9-94.5)$ & 90.6 (79.7-95.9) & $9.28(3.99-21.53)$ & $0.14(0.06-0.32)$ & $87.5(73.9-94.5)$ & 90.6 (79.7-95.9) \\
\hline 40 & $70.0(54.6-81.9)$ & $94.3(84.6-98.1)$ & $12.37(4.04-37.81)$ & $0.32(0.20-0.51$ & $90.3(75.1-96.7)$ & $80.6(69.1-88.6)$ \\
\hline 45 & $67.5(52.0-79.9)$ & $94.3(84.6-98.1)$ & $11.93(3.89-36.55)$ & $0.34(0.22-0.54)$ & $90.0(74.4-96.5)$ & $79.4(67.8-87.5)$ \\
\hline 50 & $42.5(28.5-57.8)$ & $94.3(84.6-98.1)$ & $7.51(2.36-23.87)$ & $0.61(0.46-0.81)$ & $85.0(64.0-94.8)$ & $68.5(57.1-78.0)$ \\
\hline
\end{tabular}


Finally, it must be highlighted that no single outcome measure can adequately assess asthma control. The clinical value of composite scores like ACT is limited by the lack of validation in a wider range of settings, particularly in patients with different asthma phenotypes.

The present study may have implications for clinical practice and future research. Such information could be beneficial when advising patients what to expect when deciding to escalate their medication and to employ potentially harmful drugs. Conversely, it is of critical importance to identify patients who are less responsive to steroid treatment and are at risk of developing persistent airway obstruction. These patients should be closely monitored and considered for novel anti-asthma drugs in order to prevent progression of the disease. In addition, attempts at treating by phenotype will aid in the development of a more rational approach to the evaluation of interventions like therapy with omalizumab, mepolizumab, imatimib or anti-tumor necrosis factor- $\alpha$ agents.

In conclusion, the current results suggest that FeNO can identify patients with difficult-to-treat asthma and the potential to respond to high doses of inhaled corticosteroids or systemic steroids.

\section{STATEMENT OF INTEREST}

Statements of interest for L.A. Pérez-de-Llano and for the study itself can be found at www.erj.ersjournals.com/misc/statements.dtl

\section{REFERENCES}

1 Partridge MR, van der Molen T, Myrseth SE, et al. Attitudes and actions of asthma patients on regular maintenance therapy: the INSPIRE study. BMC Pulm Med 2006; 6: 13.

2 Chapman KR, Boulet LP, Rea RM, et al. Suboptimal asthma control: prevalence, detection and consequences in general practice. Eur Respir J 2008; 31: 320-325.

3 Rabe KF, Vermeire PA, Soriano JB, et al. Clinical management of asthma in 1999: the Asthma Insights and Reality in Europe (AIRE) study. Eur Respir J 2000; 16: 802-807.

4 Bateman ED, Hurd SS, Barnes PJ, et al. Global strategy for asthma management and prevention: GINA executive summary. Eur Respir J 2008; 31: 143-178.

5 Campbell JD, Borish L, Haselkorn $\mathrm{T}$, et al. The response to combination therapy treatment regimens in severe/difficult-totreat asthma. Eur Respir J 2008; 32: 1237-1242.

6 Smith AD, Cowan JO, Brassett KP, et al. Use of exhaled nitric oxide measurements to guide treatment in chronic asthma. $N$ Engl J Med 2005; 352: 2163-2173.

7 Little SA, Chalmers GW, MacLeod KJ, et al. Non-invasive markers of airway inflammation as predictors of oral steroid responsiveness in asthma. Thorax 2000; 55: 232-234.

8 Smith $\mathrm{AD}$, Cowan JO, Brassett KP, et al. Exhaled nitric oxide: a predictor of steroid response. Am J Respir Crit Care Med 2005; 172 : 453-459.

9 Miller MR, Hankinson J, Brusasco V, et al. Standardisation of spirometry. Eur Respir J 2005; 26: 319-338.

10 Cockcroft DW, Killian DN, Mellon JJ, et al. Bronchial reactivity to inhaled histamine: a method and clinical survey. Clin Allergy 1977; 7: 235-243.
11 Vega JM, Badia X, Badiola C, et al. Validation of the Spanish version of the Asthma Control Test (ACT). J Asthma 2007; 44: 867872.

12 Nathan RA, Sorkness CA, Kosinski M, et al. Development of the asthma control test: a survey for assessing asthma control. J Allergy Clin Immunol 2004; 13: 59-65.

13 Goldberg D, Bridges K, Duncan-Jones P, et al. Detecting anxiety and depression in general medical settings. BMJ 1988; 297: 897899.

14 ROC Analysis: Web-based Calculator for ROC Curves. Baltimore, Johns Hopkins University. www.jrocfit.org Date last updated May 17, 2006. Date last accessed: July 17, 2009.

15 Jatakanon A, Lim S, Kharitonov SA, et al. Correlation between exhaled nitric oxide, sputum eosinophils, and methacholine responsiveness in patients with mild asthma. Thorax 1998; 53: 91-95.

16 Payne DN, Adcock IM, Wilson NM, et al. Relationship between exhaled nitric oxide and mucosal eosinophilic inflammation in children with difficult asthma, after treatment with oral prednisolone. Am J Respir Crit Care Med 2001; 164: 1376-1381.

17 Pavord ID, Brightling CE, Woltmann G, et al. Non-eosinophilic corticosteroid unresponsive asthma. Lancet 1999; 353: 2213-2214.

18 Meijer RJ, Postma DS, Kauffman HF, et al. Accuracy of eosinophils and eosinophil cationic protein to predict steroid improvement in asthma. Clin Exp Allergy 2002; 32: 1096-1103.

19 Kharitonov SA, Yates D, Robbins RA, et al. Nitric oxide in exhaled air of asthmatic patients. Lancet 1994; 343: 133-135.

20 Silkoff PE, Lent AM, Busacker AA, et al. Exhaled nitric oxide identifies the persistent eosinophilic phenotype in severe refractory asthma. J Allergy Clin Immunol 2005; 116: 1249-1255.

21 Little SA, Chalmers GW, MacLeod KJ, et al. Non-invasive markers of airway inflammation as predictors of oral steroid responsiveness in asthma. Thorax 2000; 55: 232-234.

22 Bateman ED, Boushey HA, Bousquet J, et al. Can guideline-defined asthma control be achieved? The Gaining Optimal Asthma Control study. Am J Respir Crit Care Med 2004; 170: 836-844.

23 Wenzel S. Severe asthma in adults. Am J Respir Crit Care Med 2005 172: $149-160$

24 The ENFUMOSA Study Group. The ENFUMOSA cross-sectional European multicentre study of the clinical phenotype of chronic severe asthma. Eur Respir J 2003; 22: 470-477.

25 Reddel HK, Taylor DR, Bateman ED, et al. An official American Thoracic Society/European Respiratory Society statement: asthma control and exacerbations: standardizing endpoints for clinical asthma trials and clinical practice. Am J Respir Crit Care Med 2009; 180: 59-99.

26 Schatz M, Mosen DM, Kosinski M, et al. Predictors of asthma control in a random sample of asthmatic patients. J Asthma 2007; 44: 341-345.

27 Robinson DS, Campbell DA, Durham SR, et al. Systematic assessment of difficult-to-treat asthma. Eur Respir J 2003; 22: 478483.

28 Heaney LG, Conway E, Kelly C, et al. Predictors of therapy resistant asthma: outcome of a systematic evaluation protocol. Thorax 2003; 58: 561-566.

29 Strine TW, Mokdad AH, Balluz LS, et al. Impact of depression and anxiety on quality of life, health behaviors, and asthma control among adults in the United States with asthma, 2006. J Asthma 2008; 45: 123-133.

30 Gamble J, Stevenson M, McClean E, et al. The prevalence of nonadherence in difficult asthma. Am J Respir Crit Care Med 2009, 180: 817-822. 